\title{
JECP/QSAED, a Computer Program for Quantification of SAED Patterns
}

\section{X.Z. Li}

\author{
Nebraska Center for Materials and Nanoscience, University of Nebraska, Lincoln, NE 68588
}

Selected-area electron diffraction (SAED) technique is one of the important analysis methods in materials science and nano-techniques. Quantitative analysis of SAED patterns, in the first step, needs to convert SAED patterns to data sets of diffraction intensities and/or lattice spacings. The SAED pattern taken from a polycrystalline sample consists of diffraction rings in concentric circles; while the one taken from a monocrystallline grain consists of diffraction spots in a periodic reciprocal lattice. Thus the quantification process of SAED patterns may be coded in software separately according to their geometric features. A computer program for the processing and quantification of the SAED ring patterns, QPCED2.0, was reported in microscopy \& microanalysis 2011 [1,2]. In the present work, a computer program for the quantification of SAED spot patterns, JECP/QSAED, has been developed.

JECP/QSAED is designed to retrieve/display the diffraction intensities and lattice spacings of SAED spot patterns, including precession electron diffraction (PED) patterns. The features of JECP/QSAED are easy to use and to get results quickly. To start the quantitation process in JECP/QSAED, user just needs to select three noncollinear diffraction spots and to index them with 2-dimensional (H K). The locations and circular areas of diffraction spots are then refined using the least-square refinement method. The background scattering for each diffraction spot is evaluated by using the intensities at the perimeter of each diffraction spot. The intensities of diffraction spots are obtained by integrating the intensity within the circular area and subtracting the background intensity. The indices of each diffraction spot can be converted from $(\mathrm{H} \mathrm{K})$ to $(\mathrm{h} \mathrm{k}$ l) with user-defined transition relation. JECP/SAED also includes other tools, e.g., a reduced cell builder for the determination of unit-cell and a linear profile scanner for the analysis of the SAED patterns taken from aperiodic structures or coexisted phases.

Figure 1 shows the graphic user interface of JECP/QSAED. An experimental SAED pattern is loaded in. Two basic spots are labeled as 1,2 and the incident beam as 3 . The reciprocal lattice spacings of the two basic spots are listed in the bottom of the frame. The positions of the diffraction spots were refined within a preset resolution limit. The intensities of the diffraction spots are retrieved and displayed in circles with various radiuses. The experimental SAED pattern is marked with a scan line in Figure 2(a) and the corresponding diffraction intensity profile is displayed with grids to show the reciprocal lattice spacing in Figure 2 (b).

JECP/QSAED was written in Java SE6. Java Runtime Environment (JRE) and Java Advanced Imaging (JAI) are required to run JECP/QSAED. The supplementary documents for JECP/QSAED can be downloaded from the author's web pages [3].

\section{References}

[1] X.Z. Li, Microsc. Microanal. 17: Suppl. S02 (2011) p. 1096.

[2] X.Z. Li, J. Appl. Cryst. 45 (2012) p. 862.

[3] http://www.unl.edu/ncmn-cfem/xzli/ and http://landyne.ueuo.com. 


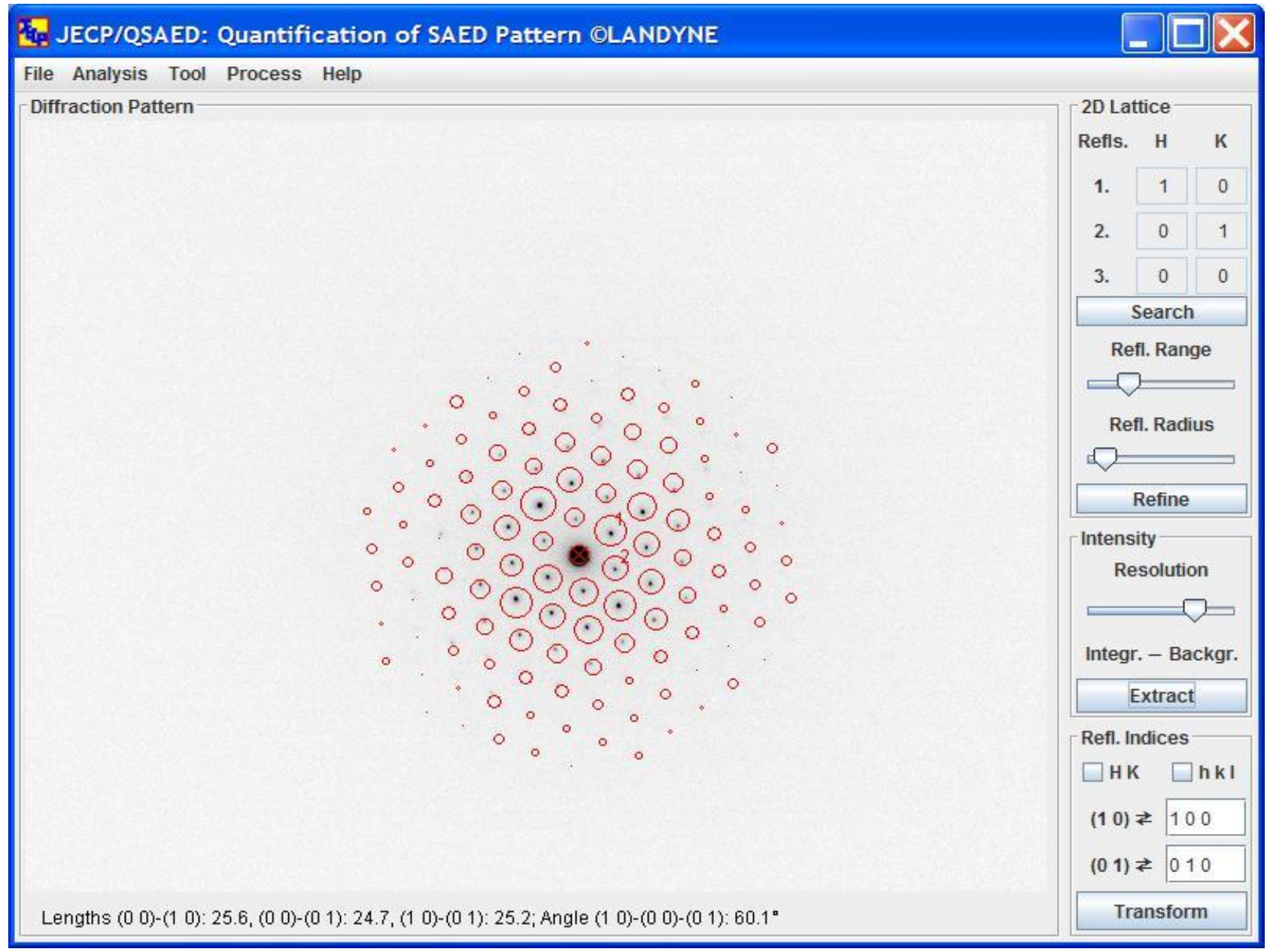

Figure 1. Graphic user interface of JECP/QSAED with an experimental SAED pattern. The intensities of diffraction spots are displayed in circles with various radiuses.

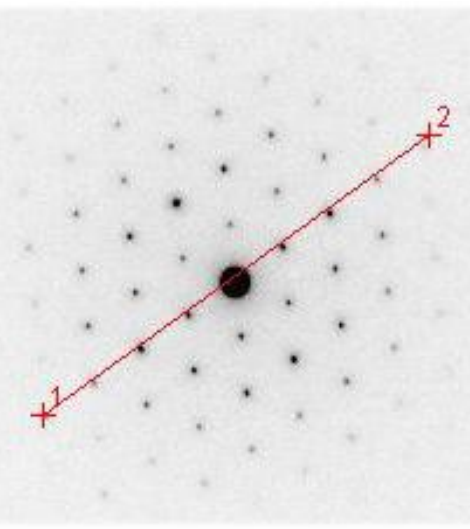

(a)

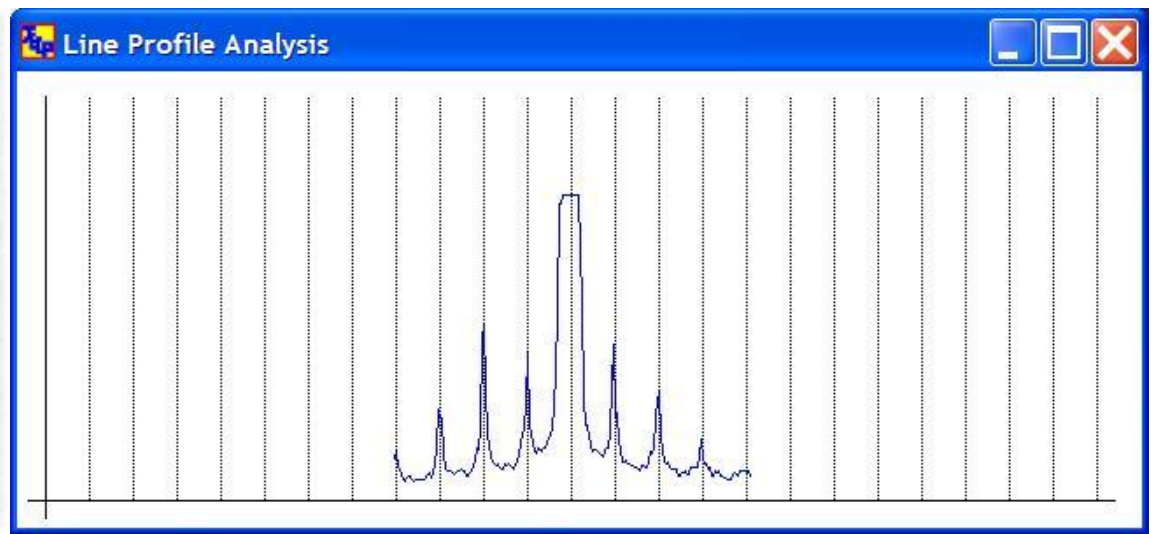

(b)

Figure 2. (a) Experimental SAED pattern with a scan line for intensity profile and (b) the corresponding diffraction intensity profile. 